\title{
Analysis of Footprint of Uncertainty of the IT2 Mamdani Controllers Using Different Type-Reducers
}

\section{Chaolong Zhang}

Central South University

\section{Haibo Zhou}

Central South University

\section{Zhiqiang Li}

Central South University

Xia Ju

Central South University

Shuaixia Tan ( $05453639 @ q q . c o m$ )

Zhuzhou Times New Material Technology CO., LTD https://orcid.org/0000-0003-1869-3709

\section{Research Article}

Keywords: footprint of uncertainty, interval type-2 fuzzy set, Mamdani fuzzy control, type-reducer

Posted Date: April 30th, 2021

DOI: https://doi.org/10.21203/rs.3.rs-395199/v1

License: (c) (1) This work is licensed under a Creative Commons Attribution 4.0 International License. Read Full License 


\title{
Analysis of Footprint of Uncertainty of the IT2 Mamdani Controllers Using Different Type-Reducers
}

\author{
Chaolong Zhang ${ }^{\mathrm{a}, \mathrm{b}}$, Haibo Zhou ${ }^{\mathrm{a}, \mathrm{b}}$, Zhiqiang $\mathrm{Li}^{\mathrm{a}, \mathrm{b}}$, Xia Ju $\mathrm{Ju}^{\mathrm{a}, \mathrm{b}}$, and Shuaixia Tan ${ }^{\mathrm{c}, *}$ \\ ${ }^{a}$ College of Mechanical and Electrical Engineering, Central South University, Changsha, Hunan 410083, China \\ ${ }^{\mathrm{b}}$ State Key Laboratory of High Performance Complex Manufacturing, Central South University, Changsha, Hunan 410083, China \\ ' Zhuzhou Times New Material Technology CO., LTD, Zhuzhou, Hunan 412007, China
}

1

Abstract-Appropriate Footprint of Uncertainties (FOUs)
are beneficial to the performance of Interval Type-2 (IT2) fuzzy
controller, revealing the effect of FOUs is a key problem. In our
published work, as the FOUs increase, the IT2 Mamdani and TS
fuzzy controllers, using KM or EKM type-reducer (TR),
approach the constant and piecewise linear controllers,
respectively, while they finally become constant and piecewise
linear controllers. To verify the validation of the above results,
when a different TR is used, in this study, the effects of other
popular TRs (i.e., Nie-Tan, Wu-Mendel, Iterative Algorithm with
Stop Condition) on output of IT2 Mamdani fuzzy controller, are
explored. We proven that, (1) as the FOUs increase, irrespectively
of the TRs used, the IT2 Mamdani fuzzy controllers approach
constant controllers, (2) when all the FOUs are equal to 1 (i.e., at
their maximum ), the fuzzy controllers using Nie-Tan and
Iterative Algorithm with Stop Condition TR become constant
controllers. The FOUs of the controllers using Wu-Mendel TR
can be infinitely approaching 1 and cannot be equal to 1
(otherwise, the denominator of the TR output expression are
equal to 0), hence when FOUs are infinitely approaching 1, the
controller will approach the constant controller infinitely. These
results imply regardless of which popular TR is used, the IT2
Mamdani fuzzy controller, when using larger FOUs, the
fluctuation of the input variables have a limited impact on the
output, the ability to deal with system uncertainties will
deteriorate. Laboratory control experiments are provided to
demonstrate these findings.
Key words- footprint of uncertainty, interval type-2 fuzzy set,
Mand Mamdani fuzzy control, type-reducer

\section{INTRODUCTION}

As one of the important components of the IT2 Fuzzy Controller (FC) (Huang et al., 2018; Zhou \& Ying, 2013, 2017), the Type-Reducer (TR) realizes the conversion of the output fuzzy set from T2 to T1, which has always attracted the interest of many researchers (Roy \& Maiti, 2020; Chen \& Wang, 2018; Li et al., 2018; Wu, 2013; Chen et al., 2018; Dongrui \& Nie, 2011; Runkler et al., 2018; Wu, 2012; Zhou et al., 2019). In (Roy \& Maiti, 2020), some new TRs, based on simple algorithm, are proposed for Stackelberg game, to solve respective large scale problems. In previous paper (Zhou et al., 2019), it was observed that, as FOUs of input fuzzy sets increase, the IT2 Mamdani and TS FCs, using mostly Karnik-Mendel (KM) or Enhanced KM (EKM) TR and min()

1 *Corresponding author: Shuaixia Tan (65453639@qq.com). operator, as well as other general configurations, approach constant and piecewise linear controllers, respectively. When FOUs are at their maximum, Mamdani FCs become constant controllers and TS FCs become piecewise linear controllers. Hence a key problem is that, it is not clear enough, when different TR is used, whether the increase of FOUs has the same effects on the IT2 FC.

At present, there are many methods of TR in the literature (Wu \& Mendel, 2009; Nie \& Tan, 2008; Melgarejo, 2007; Wu \& Mendel, 2002; Karnik et al., 1999). According to findings in the Web of Science, the most widely used methods are the KM, EKM, Nie-Tan (NT), Wu-Mendel (WM) and Iterative Algorithm with Stop Condition (IASC) TR. However, the KM TR is the most popular choice, when designing an IT2 FC. The number of papers studying TRs is still relatively low (Zhou \& Ying et al., 2019; Runkler et al., 2018; Nie \& Tan, 2012; Wu, 2012; Chen \& Wu et al., 2018; Chen \& John et al., 2018; Chen \& Wang, 2018; Li et al., 2018; Wu, 2013; Dongrui \& Nie, 2011; Wu \& Mendel, 2019). Nie and Tan studied the analytical structure of the IT2 FC, using the KM TR and the $\min ()$ operator, the controller used symmetric rule consequents, to calculate the boundary of switch points of KM TR, while, after firing intervals were determined, the analytical structure was derived (Nie \& Tan, 2012). Wu summarized the characteristics of TR methods and compared these using experiments. Nie-Tan and $\mathrm{Wu}$-Tan proved that TRs have a relatively fast response speed (Wu, 2012). Comprehensive descriptions and comparisons of the TRs are given in (Mendel et al., 2020; Chen \& Wu et al., 2018; Mendel, 2013). The speed of the algorithms depends on the coding language (Chen \& Wu et al., 2018), while the Enhanced Iterative Algorithm with Stop Condition (Dongrui \& Nie, 2011) appears to be the fastest one when running in Matlab, coded in C and Java, whereas the Enhanced KM algorithms (Wu \& Mendel, 2009) and the optimized direct approach are the fastest ones, when coded in R and Python.

In this study, based on the effects of increasing FOUs on the output of IT2 Mamdani FCs, using KM or EKM TR, the focus is mainly on, the variation of increasing FOUs on the output of IT2 Mamdani FCs, when NT, WM, and IASC TR are used. Detailed mathematical analysis provides these results: although different TRs and different fuzzy operators (i.e., product or $\min ()$ operator) are used, (1) as FOUs of input sets increase, IT2 Mamdani FCs approach constant controllers; (2) when all 
FOUs of IT2 input fuzzy sets reach their maximum (i.e., FOUs=1), the IT2 Mamdani FCs, using NT and IASC TR, become constant controllers. When FOUs are infinitely approaching 1, the controller using WM TR will infinitely approach the constant controller behavior.

In Section II, the general configuration of IT2 Mamdani FCs is described, with any number of input variables, any number and arbitrary type of IT2 fuzzy sets, two fuzzy AND operators (i.e., product and $\min ()$ ), three widely used TRs (i.e., NT, WM and IASC TRs) and centroid defuzzifier. In Section III, the effects of increasing FOUs on IT2 Mamdani FC are analyzed, when $\min ()$ operator and one of these TRs are used separately. Section IV shows the effects of the different fuzzy operators on the derived theoretical results. In Section V, real-time control experiments, in laboratory environment, are provided to inspect the theoretical properties. Finally, the derived conclusions are presented in Section VI.

\section{CONFIGURATION OF THE IT2 MAMDANI FUZZY CONTROLLER}

Let the IT2 FC have $N$ input variables, $x_{m}(1 \leq m \leq N)$, and one output variable, $u(\mathbf{x}), \mathbf{x}=\left(x_{1}, \ldots, x_{N}\right)$ represented as $u$ for simplicity reasons. Let $x_{m}$ belong to $\left[U_{m}, V_{m}\right]$, which is divided into $n_{m}-1$ subintervals: $\left[R_{m}^{1}, R_{m}^{2}\right], \ldots$, $\left[R_{m}^{k_{m}}, R_{m}^{k_{m+1}}\right], \ldots,\left[R_{m}^{n-1}, R_{m}^{n}\right], 1 \leq k_{m} \leq n_{m}$. There are $n_{m}$ IT2 fuzzy sets, dividing the range $\left[U_{m}, V_{m}\right]$ as: $P_{m}^{1}, \ldots, P_{m}^{k_{m}}, \ldots$, $P_{m}^{n_{m}} \cdot P_{m}^{k_{m}}$, defined over $\left[R_{m}^{k_{m}}, R_{m}^{k_{m+1}}\right]$, while $\theta_{m}^{k_{m}}$ represents its FOUs, as shown in Fig. 1. $\underline{\mu}_{P_{m}^{k_{m}}}\left(x_{m}, \theta_{m}^{k_{m}}\right)$ and $\bar{\mu}_{P_{m}^{k_{m}}}\left(x_{m}, \theta_{m}^{k_{m}}\right)$ are used to designate the lower and upper primary membership functions of $x_{m}$, respectively. These membership functions of input variables can be any shape, if they satisfy two following conditions: (a) $0 \leq \underline{\mu}_{P_{m}^{k_{m}}}\left(x_{m}, \theta_{m}^{k_{m}}\right) \leq \bar{\mu}_{P_{m}^{k_{m}}}\left(x_{m}, \theta_{m}^{k_{m}}\right) \leq 1$; (b) in $\left[R_{m}^{k_{m}}, R_{m}^{k_{m+1}}\right] \quad, \quad \partial \underline{\mu}_{P_{m}^{k_{m}}}\left(x_{m}, \theta_{m}^{k_{m}}\right) / \partial \theta_{m}^{k_{m}} \leq 0 \quad$ and $\partial \bar{\mu}_{P_{m}^{k_{m}}}\left(x_{m}, \theta_{m}^{k_{m}}\right) / \partial \theta_{m}^{k_{m}} \geq 0$. One can get new different controllers, if $\theta_{m}^{k_{m}}$ of the membership function is changed, thus the IT2 FC is considered as a class other than one, hence it is reasonable to consider condition (b) (Hassan et al., 2015; Yip et al., 2019). Fig.1 provides some examples of IT2 fuzzy sets.

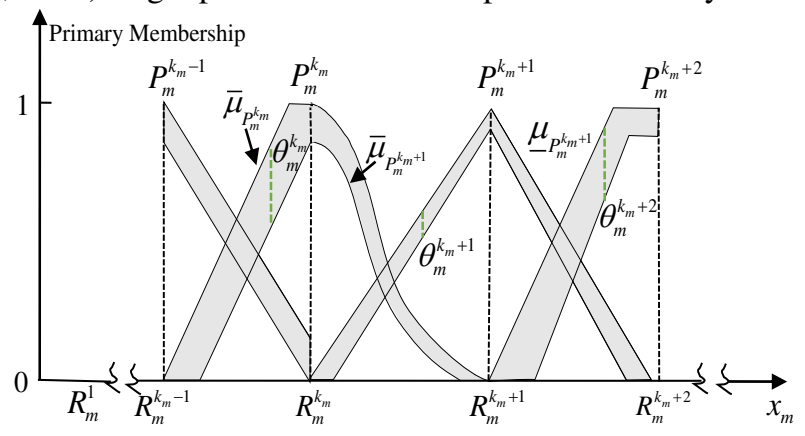

Fig. 1. Example IT2 fuzzy sets for the input variable $x_{\mathrm{m}}$.
All of $M=\prod_{m=1}^{N} n_{m}$ or less fuzzy rules are designed to represent all combinations of input fuzzy sets in IT2 FCs. For Mamdani controller, the $i$-th rule is:

IF $x_{1}$ is $P_{1}^{k_{1}}$ and $\ldots x_{N}$ is $P_{N}^{k_{N}}$, THEN $u_{i}$ is $Q_{i}$.

where $Q_{i}(1 \leq i \leq M)$ can be any continuous T2 fuzzy set. The centroid of $Q_{i}$ is assumed to represent the fuzzy set $\left[\alpha_{i}^{\prime}, \beta_{i}^{\prime}\right]$, where the terminal points $\alpha_{i}^{\prime}$ and $\beta_{i}^{\prime}$ are constants.

Product fuzzy AND operator is used to obtain firing interval, for the $i$-th rule, denoted as $f_{i}(\mathbf{x}, \theta)$ and determined as:

$$
\begin{aligned}
f_{i}(\mathbf{x}, \theta) & =\left[\underline{f}_{i}(\mathbf{x}, \theta), \bar{f}_{i}(\mathbf{x}, \theta)\right] \\
& =\left[\prod_{m=1}^{N} \underline{\mu}_{P_{m}^{k_{m}}}\left(x_{m}, \theta_{m}^{k_{m}}\right), \prod_{m=1}^{N} \bar{\mu}_{P_{m}^{k_{m}}}\left(x_{m}, \theta_{m}^{k_{m}}\right)\right]
\end{aligned}
$$

Zadeh fuzzy AND operator (i.e., $\min ($ ) operator) can also be employed to obtain firing interval, for the $i$-th rule, $f_{i}(\mathbf{x}, \theta)$, determined as:

$$
\begin{aligned}
f_{i}(\mathbf{x}, \theta) & =\left[\underline{f_{i}}(\mathbf{x}, \theta), \bar{f}_{i}(\mathbf{x}, \theta)\right] \\
& =\left[\begin{array}{l}
\min \left(\underline{\mu}_{P_{1}^{k_{1}}}\left(x_{1}, \theta_{1}^{k_{1}}\right), \ldots, \underline{\mu}_{P_{N}^{k_{N}}}\left(x_{N}, \theta_{N}^{k_{N}}\right)\right), \\
\min \left(\bar{\mu}_{P_{1}^{k_{1}}}\left(x_{1}, \theta_{1}^{k_{1}}\right), \ldots, \bar{\mu}_{P_{N}^{k_{N}}}\left(x_{N}, \theta_{N}^{k_{N}}\right)\right)
\end{array}\right]
\end{aligned}
$$

In (1) and (2), $\theta=\left(\theta_{1}^{k_{1}}, \ldots, \theta_{i}^{k_{i}}, \ldots, \theta_{N}^{k_{N}}\right)$. For convenience, $f_{i}$,

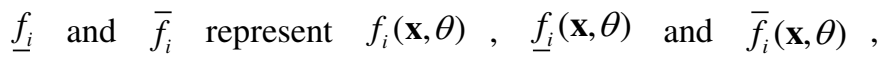
respectively.

For the IT2 FC, it is necessary to reduce the T2 output fuzzy set to a T1 fuzzy set, before defuzzification. The following three popular TRs (except KM and EKM TR) are used to obtain the output of controller, respectively.

Considering the three commonly used TRs (Nie \& Tan, 2008; Melgarejo, 2007; Wu \& Mendel, 2002), it is obvious that, whether the $\alpha_{k}$ and $\beta_{k}$ are sorted, has no effect on the NT TR and the WM TR, but the IASC TR requires the $\alpha_{k}$ and $\beta_{k}$ to be sorted in ascending order. For the convenience of description, all $\alpha_{k}$ and $\beta_{k}$ are unified, arranged in ascending order, $\alpha_{1} \leq \ldots \leq \alpha_{k} \leq \ldots \leq \alpha_{M}$ and $\beta_{1} \leq \ldots \leq \beta_{k} \leq \ldots \leq \beta_{M}$, while $\underline{f}_{k}$ is also arranged in the same order as $\alpha_{k}$ and $\bar{f}_{k}$ as $\beta_{k}$. The resulting left and right points of the firing intervals are written as $\underline{f}_{k}^{*}$ and $\bar{f}_{k}^{*}$, respectively.

The output of NT TR is an accurate closed-form solution, so there is no need to perform defuzzification process. The output of the IT2 FC is as follows:

$$
u(\mathbf{x}, \theta)=\frac{\sum_{k=1}^{M} u_{k}\left(\bar{f}_{k}^{*}+\underline{f}_{k}^{*}\right)}{\sum_{k=1}^{M}\left(\bar{f}_{k}^{*}+\underline{f}_{k}^{*}\right)}
$$

Note that the rule consequents $u_{k}$ of IT2 FC, using the NT TR, do not need to be sorted, since $u_{k}=\left(\alpha_{k}+\beta_{k}\right) / 2$.

Wu and Mendel proposed a WM TR controller (also known 
as Uncertainty Bound TR) that can effectively reduce the computational burden (Wu \& Mendel, 2002), mainly approximating the output $\left[u_{l}^{K M}(\mathbf{x}, \theta), u_{r}^{K M}(\mathbf{x}, \theta)\right]$ of KM TR, through uncertain boundaries. In the case of IT2 Mamdani FC, WM TR uses a four-steps iterative process:

(1) Computation of the boundary T1 fuzzy sets for the employed IT2 fuzzy sets:

$$
\begin{aligned}
& \bar{u}_{l}^{(1)}(\mathbf{x}, \theta)=\frac{\sum_{k=1}^{M} \underline{f}_{k}^{*} \alpha_{k}}{\sum_{k=1}^{M} \underline{f}_{k}^{*}} \\
& \bar{u}_{l}^{(2)}(\mathbf{x}, \theta)=\frac{\sum_{k=1}^{M} \bar{f}_{k}^{*} \alpha_{k}}{\sum_{k=1}^{M} \bar{f}_{k}^{*}} \\
& \underline{u}_{r}^{(1)}(\mathbf{x}, \theta)=\frac{\sum_{k=1}^{M} \underline{f}_{k}^{*} \beta_{k}}{\sum_{k=1}^{M} \underline{f}_{k}^{*}} \\
& \underline{u}_{r}^{(2)}(\mathbf{x}, \theta)=\frac{\sum_{k=1}^{M} \bar{f}_{k}^{*} \beta_{k}}{\sum_{k=1}^{M} \bar{f}_{k}^{*}}
\end{aligned}
$$

(2) Calculation of the suitable value of $\bar{u}_{l}(\mathbf{x}, \theta)$ and $\underline{u}_{r}(\mathbf{x}, \theta)$ through:

$$
\begin{aligned}
& \bar{u}_{l}(\mathbf{x}, \theta)=\min \left[\bar{u}_{l}^{(1)}(\mathbf{x}, \theta), \bar{u}_{l}^{(2)}(\mathbf{x}, \theta)\right] \\
& \underline{u}_{r}(\mathbf{x}, \theta)=\max \left[\underline{u}_{r}^{(1)}(\mathbf{x}, \theta), \underline{u}_{r}^{(2)}(\mathbf{x}, \theta)\right]
\end{aligned}
$$

(3) Computation of $\underline{u}_{l}(\mathbf{x}, \theta)$ and $\bar{u}_{r}(\mathbf{x}, \theta)$ as:

$$
\begin{aligned}
& \underline{u}_{l}(\mathbf{x}, \theta)=\bar{u}_{l}(\mathbf{x}, \theta) \\
& -\frac{\sum_{k=1}^{M}\left(\bar{f}_{k}^{*}-\underline{f}_{k}^{*}\right)}{\sum_{k=1}^{M} \bar{f}_{k}^{*} \times \sum_{k=1}^{M} \underline{f}_{k}^{*}} \times \frac{\sum_{k=1}^{M} \underline{f}_{k}^{*}\left(\alpha_{k}-\alpha_{1}\right) \times \sum_{k=1}^{M} \bar{f}_{k}^{*}\left(\alpha_{M}-\alpha_{k}\right)}{\sum_{k=1}^{M} \underline{f}_{k}^{*}\left(\alpha_{k}-\alpha_{1}\right)+\sum_{k=1}^{M} \bar{f}_{k}^{*}\left(\alpha_{M}-\alpha_{k}\right)} \\
& \bar{u}_{r}(\mathbf{x}, \theta)=\underline{u}_{r}(\mathbf{x}, \theta) \\
& +\frac{\sum_{k=1}^{M}\left(\bar{f}_{k}^{*}-\underline{f}_{k}^{*}\right)}{\sum_{k=1}^{M} \bar{f}_{k}^{*} \times \sum_{k=1}^{M} \underline{f}_{k}^{*}} \times \frac{\sum_{k=1}^{M} \bar{f}_{k}^{*}\left(\beta_{k}-\beta_{1}\right) \times \sum_{k=1}^{M} \underline{f}_{k}^{*}\left(\beta_{M}-\beta_{k}\right)}{\sum_{k=1}^{M} \bar{f}_{k}^{*}\left(\beta_{k}-\beta_{1}\right)+\sum_{k=1}^{M} \underline{f}_{k}^{*}\left(\beta_{M}-\beta_{k}\right)}
\end{aligned}
$$

\section{(4) Computation of}

$$
\begin{aligned}
& u_{l}(\mathbf{x}, \theta)=\left(\bar{u}_{l}(\mathbf{x}, \theta)+\underline{u}_{l}(\mathbf{x}, \theta)\right) / 2 \\
& u_{r}(\mathbf{x}, \theta)=\left(\bar{u}_{r}(\mathbf{x}, \theta)+\underline{u}_{r}(\mathbf{x}, \theta)\right) / 2
\end{aligned}
$$

Unlike the KM TR, the $\alpha_{i}$ and $\beta_{i}$ do not need to be sorted, but their maximum and minimum values need to be specified. For convenience, it is assumed $\alpha_{1} \leq \alpha_{i} \leq \alpha_{M}, \beta_{1} \leq \beta_{i} \leq \beta_{M}$, $i=1, \ldots, M$.

In the case of IASC TR (Melgarejo, 2007), iterative termination conditions are used to effectively reduce the computational burden of the KM TR. The iteration process produces the final output of IASC TR as:

$$
\begin{aligned}
& u_{l}(\mathbf{x}, \theta)=\frac{\sum_{k=1}^{M} \underline{f}_{k}^{*} \alpha_{k}+\sum_{k=1}^{P_{L}}\left(\bar{f}_{k}^{*}-\underline{f}_{k}^{*}\right) \alpha_{k}}{\sum_{k=1}^{M} \underline{f}_{k}^{*}+\sum_{k=1}^{P_{L}}\left(\bar{f}_{k}^{*}-\underline{f}_{k}^{*}\right)} \\
& u_{r}(\mathbf{x}, \theta)=\frac{\sum_{k=1}^{M} \bar{f}_{k}^{*} \beta_{k}-\sum_{k=1}^{P_{R}}\left(\bar{f}_{k}^{*}-\underline{f}_{k}^{*}\right) \beta_{k}}{\sum_{k=1}^{M} \bar{f}_{k}^{*}-\sum_{k=1}^{P_{R}}\left(\bar{f}_{k}^{*}-\underline{f}_{k}^{*}\right)}
\end{aligned}
$$

It should be note that the rule consequents $\alpha_{k}$ and $\beta_{k}$ are required to be sorted in ascending order.

In the case where the WM or IASC TR is used, the output is a T1 fuzzy set $\left[u_{l}(\mathbf{x}, \theta), u_{r}(\mathbf{x}, \theta)\right]$, instead of direct output of the closed solution $u(\mathbf{x}, \theta)$ as NT TR, thus the centroid defuzzification needs to be applied, in order to obtain the accurate closed solution:

$$
u(\mathbf{x}, \theta)=\frac{1}{2}\left(u_{l}(\mathbf{x}, \theta)+u_{r}(\mathbf{x}, \theta)\right)
$$

Noted that for any kind of TR, $u(\mathbf{x}, \theta)$ is a $N$-dimensional surface, while after the FOUs are determined, the output $u(\mathbf{x}, \theta)$ is only related to $N$ input variables.

\section{EFFECTS OF THE FOUS ON THE OUTPUT OF THE IT2 MAMDANI FUZZY CONTROLLERS}

In this Section, the $\min ()$ operator is used to obtain the firing intervals, while the effects of increasing the FOUs, on the output of the IT2 Mamdani FCs, are researched, in the respective cases of NT, WM, and IASC TR use. When the FOUs of all input IT2 fuzzy sets are at their maximum, the following are respectively denoted: $u^{N T}(\mathbf{x}, \theta)$ as $U^{N T}(\mathbf{x}, \theta)$, $u^{W M}(\mathbf{x}, \theta)$ as $U^{W M}(\mathbf{x}, \theta), u^{I A S C}(\mathbf{x}, \theta)$ as $U^{I A S C}(\mathbf{x}, \theta)$.

\section{A. Use of NT type-reducer}

In order to demonstrate the effects of increasing FOU on the controller, using NT TR, three properties are summarized as follows:

Property 1: When the FOUs are at their maximum, the IT2 Mamdani FC, using the NT TR, becomes constant controller.

Proof: When the FOUs are at their maximum and $\theta=1, \bar{\mu}_{k}=1, \underline{\mu}_{k}=0$, i.e., $\bar{f}_{k}^{*}=1, \underline{f}_{k}^{*}=0$, the following relation applies:

$$
u^{N T}(\mathbf{x}, \theta)=\frac{\sum_{k=1}^{M} u_{k}\left(\bar{f}_{k}^{*}+\underline{f}_{k}^{*}\right)}{\sum_{k=1}^{M}\left(\bar{f}_{k}^{*}+\underline{f}_{k}^{*}\right)}=\frac{\sum_{k=1}^{M} u_{k}}{M}
$$

Therefore, when the FOUs reach their maximum, no matter what the input variables are, the output is always constant, that is, the controller becomes a constant controller.

QED 


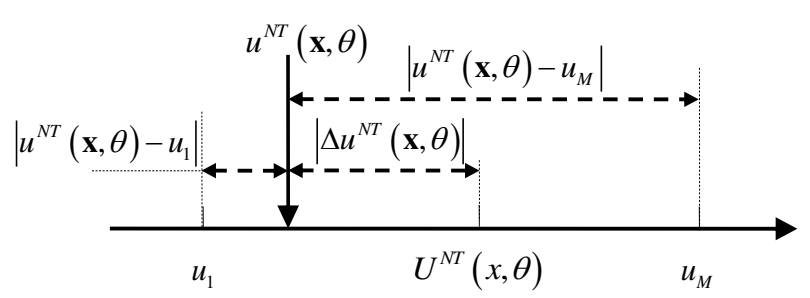

Fig. 2. An illustration of the difference between $\left|\Delta u^{N T}(\mathbf{x}, \theta)\right|$ and $\left|u^{N T}(\mathbf{x}, \theta)-u_{1}\right|+\left|u^{N T}(\mathbf{x}, \theta)-u_{M}\right|$.

Property 2: As FOUs increase, $u^{N T}(\mathbf{x}, \theta)$ gradually approaches the plane $U^{N T}(\mathbf{x}, \theta)$, while the output of the IT2 Mamdani controller, using the NT TR, is increasingly similar to a constant.

Proof: a) Convert the NT TR formula into a form similar to KM TR, as follows:

$$
\begin{aligned}
& u^{N T}(\mathbf{x}, \theta)=\frac{\sum_{k=1}^{M} u_{k}\left(\bar{f}_{k}^{*}+\underline{f}_{k}^{*}\right)}{\sum_{k=1}^{M}\left(\bar{f}_{k}^{*}+\underline{f}_{k}^{*}\right)} \\
& =\frac{\sum_{k=1}^{P_{L}} u_{k}\left(\bar{f}_{k}^{*}\right)+\sum_{k=P_{L}+1}^{M} u_{k}\left(\underline{f}_{k}^{*}\right)+\sum_{k=1}^{P_{R}} u_{k}\left(\underline{f}_{k}^{*}\right)+\sum_{k=P_{R}+1}^{M} u_{k}\left(\bar{f}_{k}^{*}\right)}{\sum_{k=1}^{P_{L}}\left(\bar{f}_{k}^{*}\right)+\sum_{k=P_{L}+1}^{M}\left(\underline{f}_{k}^{*}\right)+\sum_{k=1}^{P_{R}}\left(\underline{f}_{k}^{*}\right)+\sum_{k=P_{R}+1}^{M}\left(\bar{f}_{k}^{*}\right)}
\end{aligned}
$$

where $1 \leq P_{L}=P_{R} \leq M$.

b) Let $\Delta u^{N T}(\mathbf{x}, \theta)=\left|u^{N T}(\mathbf{x}, \theta)-U^{N T}\right|$ and consider simplified expressions, derived from Fig. 2, $\left|\Delta u^{N T}(\mathbf{x}, \theta)\right|=\left|u^{N T}(\mathbf{x}, \theta)-U^{N T}\right| \leq\left|u_{M}-u_{1}\right| \quad$, hence $0 \leq\left|\Delta u^{N T}(\mathbf{x}, \theta)\right| \leq\left|u_{M}-u_{1}\right|, \quad\left|\Delta u^{N T}(\mathbf{x}, \theta)\right| \quad$ is $\quad$ a bounded function.

An inequality is established as follows:

$$
\begin{aligned}
\left|\Delta u^{N T}(\mathbf{x}, \theta)\right| \leq A\left|\frac{\sum_{k=1}^{P_{L}} u_{k}\left(\bar{f}_{k}^{*}\right)+\sum_{k=P_{L}+1}^{M} u_{k}\left(\underline{f}_{k}^{*}\right)}{\sum_{k=1}^{P_{L}}\left(\bar{f}_{k}^{*}\right)+\sum_{k=P_{L}+1}^{M}\left(\underline{f}_{k}^{*}\right)}-u_{1}\right| \\
+B\left|\frac{\sum_{k=1}^{P_{R}} u_{k}\left(\underline{f}_{k}^{*}\right)+\sum_{k=P_{R}+1}^{M} u_{k}\left(\bar{f}_{k}^{*}\right)}{\sum_{k=1}^{P_{R}}\left(\underline{f}_{k}^{*}\right)+\sum_{k=P_{R}+1}^{M}\left(\bar{f}_{k}^{*}\right)}-u_{M}\right|
\end{aligned}
$$

c) Combine the proof process of KM TR, as described in the work published in (Zhou et al., 2019).

In the case of the IT2 Mamdani FC, using KM TR, as the FOUs increase, the following (20) and (21) are either decreasing in a monotonic or non-increase manner, becoming equal to 0 , when all the FOUs arrive at their maximum.

$$
\begin{aligned}
& \left|\Delta u_{l}^{K M}(\mathbf{x}, \theta)\right|=\left|u_{l}^{K M}(\mathbf{x}, \theta)-\alpha_{1}\right| \\
& =\left|\left(\sum_{k=1}^{P_{L}} \bar{f}_{k}^{*} \alpha_{k}+\sum_{k=P_{L}+1}^{M} \underline{f}_{k}^{*} \alpha_{k}\right) /\left(\sum_{k=1}^{P_{L}} \bar{f}_{k}^{*}+\sum_{k=P_{L}+1}^{M} \underline{f}_{k}^{*}\right)-\alpha_{1}\right|
\end{aligned}
$$

$$
\begin{aligned}
& \left|\Delta u_{r}^{K M}(\mathbf{x}, \theta)\right|=\left|u_{r}^{K M}(\mathbf{x}, \theta)-\beta_{M}\right| \\
& =\left|\left(\sum_{k=1}^{P_{R}} \underline{f}_{k}^{*} \beta_{k}+\sum_{k=P_{R}+1}^{M} \bar{f}_{k}^{*} \beta_{k}\right) /\left(\sum_{k=1}^{P_{R}} \underline{f}_{k}^{*}+\sum_{k=P_{R}+1}^{M} \bar{f}_{k}^{*}\right)-\beta_{M}\right|
\end{aligned}
$$

For FCs, the output is greater than the minimum value of rule consequents (i.e., $\alpha_{1}$ ) and less than the maximum value (i.e., $\left.\beta_{M}\right)$. Therefore, $\left|\Delta u_{l}^{K M}(\mathbf{x}, \theta)\right|$ and $\left|\Delta u_{r}^{K M}(\mathbf{x}, \theta)\right|$ are bounded functions, greater than or equal to $0\left(\left|\Delta u_{l}^{K M}(\mathbf{x}, \theta)\right|=0\right.$ and $\left|\Delta u_{r}^{K M}(\mathbf{x}, \theta)\right|=0$ only when all $\left.\theta=1\right)$. According to Theorem 1 in (Zhou et al., 2019) $\left|\Delta u^{N T}(\mathbf{x}, \theta)\right|=0$ when all $\theta=1$. Therefore, if $A B \neq 0$, the left and right ends of the (19) will become 0 , at all $\theta=1$. In other cases, $\left|\Delta u^{N T}(\mathbf{x}, \theta)\right|,\left|\Delta u_{l}^{K M}(\mathbf{x}, \theta)\right|$ and $\left|\Delta u_{r}^{K M}(\mathbf{x}, \theta)\right|$ are all bounded functions greater than 0 . Hence, for (19), there are $A$ and $B$ to satisfy the relation in Constant.

According to the study presented in (Zhou et al., 2019), as the FOUs increase, $\left|\Delta u_{l}^{K M}(\mathbf{x}, \theta)\right|$ and $\left|\Delta u_{r}^{K M}(\mathbf{x}, \theta)\right|$ are either decreasing in a monotonic fashion or non-increase, becoming 0 when all the FOUs reach their maximum. Hence, the right side of (19) approaches 0 in a monotonic decreasing or non-increasing behavior, as the FOUs increase. Also, the $\left|\Delta u^{N T}(\mathbf{x}, \theta)\right|$ become smaller and smaller, while $\left|\Delta u^{N T}(\mathbf{x}, \theta)\right|=0$, when all FOUs reach their maximum. At that time, $u^{N T}(\mathbf{x}, \theta)=\left(\sum_{k=1}^{M} u_{k}\right) / M$, the IT2 Mamdani controllers, using NT TR, become constant controllers. QED

Property 3: When $0<\theta<1$ and the derivatives of the upper and lower membership functions about $\theta$, are opposite to each other, increasing FOUs have no effect on $u^{N T}(\mathbf{x}, \theta)$.

Proof: Derivation of the output, in (3), of NT TR with respect to $\theta$ :

$$
\frac{\partial u^{N T}(\mathbf{x}, \theta)}{\partial \theta}=\frac{\left(\frac{\partial \bar{f}_{i}^{*}}{\partial \theta_{i}}+\frac{\partial \underline{f}_{i}^{*}}{\partial \theta_{i}}\right) \times \sum_{k=1}^{M}\left(\bar{f}_{k}^{*}+\underline{f}_{k}^{*}\right) \times\left(u_{i}-u_{k}\right)}{\left[\sum_{k=1}^{M}\left(\bar{f}_{k}^{*}+\underline{f}_{k}^{*}\right)\right]^{2}}
$$

Thus, when $\partial \bar{f}_{i}^{*} / \partial \theta_{i}=-\partial \underline{f}_{i}^{*} / \partial \theta_{i}, \partial u^{N T}(\mathbf{x}, \theta) / \partial \theta=0$. It should be noted that, the $u^{N T}(\mathbf{x}, \theta)$ becomes $U^{N T}(\mathbf{x}, \theta)$, when all FOUs become 1 .

QED

Considering that this situation often occurs, when the fuzzy set of the IT2 fuzzy PI and PD controller is configured (Long et al., 2019; Nie \& Tan, 2012; Du \& Ying, 2010), researchers should avoid the use of NT TR, when designing such controllers.

\section{B. Use of WM type-reducer}

In the case where WM TR is used, as FOUs increase, the output of IT2 FC changes as follows: 
Property 4: When all FOUs are infinitely approaching their maximum, the IT2 Mamdani FC, using WM TR, approximates a constant controller infinitely.

Proof: when $\theta=1, \bar{\mu}_{k}=1, \underline{\mu}_{k}=0, \bar{f}_{k}^{*}=1, \underline{f}_{k}^{*}=0$, the denominator of the (4), (6), (10) and (11) is 0 . Taking this into account, the method of taking the limit to prove is as follows:

$$
\begin{aligned}
& \lim _{\theta \rightarrow 1} \vec{u}_{l}^{-(1)}(\mathbf{x}, \theta)=\frac{\sum_{k=1}^{M} f_{k}^{*} \alpha_{k}}{\sum_{k=1}^{M} \underline{f}_{k}^{*}}=\frac{f^{*} \sum_{k=1}^{M} \alpha_{k}}{M \times \underline{f}^{*}}=\frac{\sum_{k=1}^{M} \alpha_{k}}{M} \\
& \lim _{\theta \rightarrow 1} \bar{u}_{l}^{(2)}(\mathbf{x}, \theta)=\frac{\sum_{k=1}^{M} \bar{f}_{k}^{*} \alpha_{k}}{\sum_{k=1}^{M} \bar{f}_{k}^{*}}=\frac{\sum_{k=1}^{M} \alpha_{k}}{M} \\
& \lim _{\theta \rightarrow 1} \underline{u}_{r}^{(1)}(\mathbf{x}, \theta)=\frac{\sum_{k=1}^{M} \underline{f}_{k}^{*} \beta_{k}}{\sum_{k=1}^{M} \underline{f}_{k}^{*}}=\frac{\sum_{k=1}^{M} \beta_{k}}{M} \\
& \lim _{\theta \rightarrow 1} \underline{u}_{r}^{(2)}(\mathbf{x}, \theta)=\frac{\sum_{k=1}^{M} \bar{f}_{k}^{*} \beta_{k}}{\sum_{k=1}^{M} \bar{f}_{k}^{*}}=\frac{\sum_{k=1}^{M} \beta_{k}}{M} \\
& u_{l}(\mathbf{x}, \theta)=\bar{u}_{l}(\mathbf{x}, \theta) \\
& -\frac{1}{2} \frac{\sum_{k=1}^{M}\left(\bar{f}_{k}^{*}-\underline{f}_{k}\right)}{\sum_{k=1}^{M} \bar{f}_{k}^{*} \times \sum_{k=1}^{M} \underline{f}_{k}} \times \frac{\sum_{k=1}^{M} \underline{f}_{k}^{*}\left(\alpha_{k}-\alpha_{1}\right) \times \sum_{k=1}^{M} \bar{f}_{k}^{*}\left(\alpha_{M}-\alpha_{k}\right)}{\sum_{k=1}^{M} \underline{f}_{k}^{*}\left(\alpha_{k}-\alpha_{1}\right)+\sum_{k=1}^{M} \bar{f}_{k}^{*}\left(\alpha_{M}-\alpha_{k}\right)} \\
& =\lim _{\theta \rightarrow 1}\left(\frac{\sum_{k=1}^{M} \alpha_{k}}{M}-\frac{1}{2} \frac{M}{M \times M \times f_{-}^{*}} \times \frac{\underline{f}^{*} \times \sum_{k=1}^{M}\left(\alpha_{k}-\alpha_{1}\right) \times \sum_{k=1}^{M}\left(\alpha_{M}-\alpha_{k}\right)}{\sum_{k=1}^{M}\left(\alpha_{M}-\alpha_{k}\right)}\right) \\
& =\frac{\sum_{k=1}^{M}\left(\alpha_{k}+\alpha_{1}\right)}{2 M}\left(\geq \alpha_{1}\right) \\
& u_{r}(\mathbf{x}, \theta)=\underline{u}_{r}(\mathbf{x}, \theta) \\
& +\frac{1}{2} \frac{\sum_{k=1}^{M}\left(\bar{f}_{k}^{*}-\underline{f}_{k}^{*}\right)}{\sum_{k=1}^{M} \bar{f}_{k}^{*} \times \sum_{k=1}^{M} \underline{f}_{k}^{*}} \times \frac{\sum_{k=1}^{M} \bar{f}_{k}^{*}\left(\beta_{k}-\beta_{1}\right) \times \sum_{k=1}^{M} \underline{f}_{k}^{*}\left(\beta_{M}-\beta_{k}\right)}{\sum_{k=1}^{M} \bar{f}_{k}^{*}\left(\beta_{k}-\beta_{1}\right)+\sum_{k=1}^{M} \underline{f}_{k}^{*}\left(\beta_{M}-\beta_{k}\right)} \\
& =\lim _{\theta \rightarrow 1}\left(\begin{array}{l}
\frac{\sum_{k=1}^{M} \beta_{k}}{M}+\frac{1}{2} \frac{M}{M \times M \times \underline{f}^{*}} \\
\times \frac{\sum_{k=1}^{M}\left(\beta_{k}-\beta_{1}\right) \times \underline{f}^{*} \times \sum_{k=1}^{M}\left(\beta_{M}-\beta_{k}\right)}{\sum_{k=1}^{M}\left(\beta_{k}-\beta_{1}\right)}
\end{array}\right)
\end{aligned}
$$

where $\alpha_{1}$ is the minimum value of the $\alpha_{k}(k=1 \ldots M), \beta_{M}$ is the maximum value of the $\beta_{k}(k=1 \ldots M)$, hence $u_{l} \geq \alpha_{1}, u_{r} \leq \beta_{M}$.

$$
\begin{aligned}
& U^{W M}(\mathbf{x}, \theta)=\frac{1}{2}\left(u_{l}(\mathbf{x}, \theta)+u_{r}(\mathbf{x}, \theta)\right) \\
& =\frac{\sum_{k=1}^{M}\left(\alpha_{k}+\alpha_{1}\right)}{4 M}+\frac{\sum_{k=1}^{M}\left(\beta_{M}+\beta_{k}\right)}{4 M}=\frac{\sum_{k=1}^{M}\left(\alpha_{k}+\alpha_{1}+\beta_{M}+\beta_{k}\right)}{4 M}
\end{aligned}
$$

Therefore, when $\theta$ is extremely close to 1 , the output $u^{W M}(\mathbf{x}, \theta)$ will approach the constant infinitely, that is, the IT2 Mamdani FC, using WM TR, behaves approximately as a constant controller.

QED

Property 5: As the FOUs increase, the output $u^{W M}(\mathbf{x}, \theta)$ of IT2 Mamdani FC, using WM TR, tends to remain constant.

Proof: According to Section IV of (Wu \& Mendel, 2002), the difference $|\delta(x)|$, between the output of WM TR and KM TR, uses the Risk Function to ensure that it is sufficiently small. The definition of Risk Function is as follows:

$$
R=w \times R_{T R}+(1-w) \times\left(\frac{1}{n} \sum_{m=1}^{n} \delta^{2}\left(x_{m}\right)\right)
$$

where $w$ is the weighting factor, $R_{T R}=\frac{1}{n} \sum_{m=1}^{n}\left[u^{m}-\frac{u_{l}^{m}+u_{r}^{m}}{2}\right]$ is calculated by the KM TR.

Hence,

$$
\begin{aligned}
& \left|u^{W M}(\mathbf{x}, \theta)-u^{K M}(\mathbf{x}, \theta)\right| \leq|\delta(\mathbf{x}, \theta)| \\
& \left|u^{W M}(\mathbf{x}, \theta)-u^{K M}(\mathbf{x}, \theta)\right| \\
& =\left|\left(u^{W M}(\mathbf{x}, \theta)-U^{K M}(\mathbf{x}, \theta)\right)-\left(u^{K M}(\mathbf{x}, \theta)-U^{K M}(\mathbf{x}, \theta)\right)\right| \\
& \geq\left|u^{W M}(\mathbf{x}, \theta)-U^{K M}(\mathbf{x}, \theta)\right|-\left|u^{K M}(\mathbf{x}, \theta)-U^{K M}(\mathbf{x}, \theta)\right| \\
& =\left|u^{W M}(\mathbf{x}, \theta)-U^{K M}(\mathbf{x}, \theta)\right|-\left|\Delta u^{K M}(\mathbf{x}, \theta)\right| \\
& \left|u^{W M}(\mathbf{x}, \theta)-U^{K M}(\mathbf{x}, \theta)\right|-\left|\Delta u^{K M}(\mathbf{x}, \theta)\right| \leq|\delta(\mathbf{x}, \theta)| \\
& \left|u^{W M}(\mathbf{x}, \theta)-U^{K M}(\mathbf{x}, \theta)\right| \leq|\delta(\mathbf{x}, \theta)|+\left|\Delta u^{K M}(\mathbf{x}, \theta)\right|
\end{aligned}
$$

where $\left|\Delta u^{K M}(\mathbf{x}, \theta)\right|$ approaches 0 ; as the FOUs increase, $\left|\Delta u^{K M}(\mathbf{x}, \theta)\right|$ becomes 0 when the FOUs reach their maximum. WM TR has used the Risk Function to ensure that $|\delta(\mathbf{x}, \theta)|$ is extremely small, as described in (Wu \& Mendel, 2002), otherwise it would be meaningless for $u^{W M}(\mathbf{x}, \theta)$ to approach $u^{K M}(\mathbf{x}, \theta)$. Therefore, as the FOUs increase, $\left|\Delta u^{K M}(\mathbf{x}, \theta)\right|$ gradually becomes 0 , hence $\left|u^{W M}(\mathbf{x}, \theta)-U^{K M}(\mathbf{x}, \theta)\right|$ also gradually decreases. Consequently, as the FOUs increase, the IT2 Mamdani FCs, using WM TR, gradually become similar to constant controllers.

QED 


\section{Use of IASC type-reducer}

As the FOUs increase, the output of the controller, using IASC TR, changes as follows:

Property 6: When FOUs are at their maximum, the IT2 Mamdani FC, using IASC TR, becomes a constant controller.

Proof: First, the formula of IASC TR is converted into the form of KM TR.

$$
\begin{aligned}
& u_{l}^{I A S C}(\mathbf{x}, \theta)=\frac{\sum_{k=1}^{M} \underline{f}_{k}^{*} \alpha_{k}+\sum_{k=1}^{P_{L}}\left(\bar{f}_{k}^{*}-\underline{f}_{k}^{*}\right) \alpha_{k}}{\sum_{k=1}^{M} \underline{f}_{k}^{*}+\sum_{k=1}^{P_{L}}\left(\bar{f}_{k}^{*}-\underline{f}_{k}^{*}\right)} \\
& =\frac{\sum_{k=1}^{P_{L}} \bar{f}_{k}^{*} \alpha_{k}+\sum_{k=P_{L}+1}^{M} \underline{f}_{k}^{*} \alpha_{k}}{\sum_{k=1}^{P_{L}} \bar{f}_{k}^{*}+\sum_{k=P_{L}+1}^{M} \underline{f}_{k}^{*}}=u_{l}^{K M} \\
& u_{r}^{I A S C}(\mathbf{x}, \theta)=\frac{\sum_{k=1}^{M} \bar{f}_{k}^{*} \beta_{k}-\sum_{k=1}^{P_{R}}\left(\bar{f}_{k}^{*}-\underline{f}_{k}^{*}\right) \beta_{k}}{\sum_{k=1}^{M} \bar{f}_{k}^{*}-\sum_{k=1}^{P_{R}}\left(\bar{f}_{k}^{*}-\underline{f}_{k}^{*}\right)} \\
& =\frac{\sum_{k=1}^{P_{R}} \underline{f}_{k}^{*} \beta_{k}+\sum_{k=P_{R}+1}^{M} \bar{f}_{k}^{*} \beta_{k}}{\sum_{k=1}^{P_{R}} \underline{f}_{k}^{*}+\sum_{k=P_{R}+1}^{M} \bar{f}_{k}^{*}}=u_{r}^{K M}
\end{aligned}
$$

It is derived that, the output equations of controller, using ISAC TR, are the same as those of one using KM TR; so it inherits the conclusion that the IT2 controller, using KM TR, becomes a constant controller, when FOUs are at maximum. The process for proving this conclusion is similar to the case of using KM TR, so it will not be repeated here.

QED

Property 7: As the FOUs increase, $u^{I A S C}(\mathbf{x}, \theta)$ gradually approaches the plane $\left(\alpha_{1}+\beta_{M}\right) / 2$, while the IT2 Mamdani FC is more and more similar to a constant controller.

Proof: According to (35) and (36), after the output of IASC TR is converted into a form, there is no difference from the output of KM TR. Since the output of the IT2 controller, using KM TR, is increasingly similar to the constant controller, as the FOUs increase, the IT2 controller, using IASC TR, follows also the same trend. Due to limited space, the steps of proof will not be repeated here.

QED

According to the above analysis, as FOUs increase, the output of the IT2 controller, either using NT TR, WM TR, or IASC TR, is consistent with the controller using KM and EKM TR. In order to better illustrate the effects of increasing FOUs on the output of IT2 Mamdani FC, using different TRs, their respective output surface is shown in Fig. 3. It is evident that, although the control surfaces are slightly different, when the controller uses the four TRs, the output surfaces gradually tend to a constant plane, as the FOUs increase. Furthermore, when $\theta=0.9$, the output is almost a constant plane, using any TR.

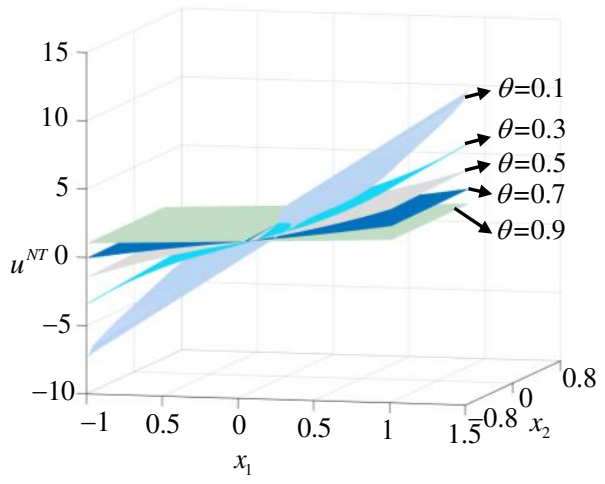

(a)

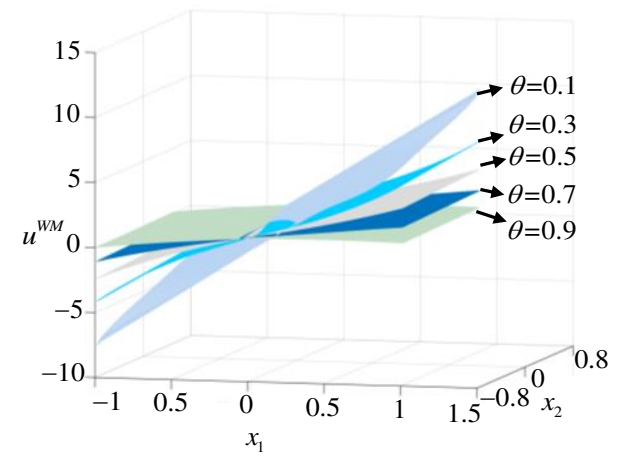

(b)

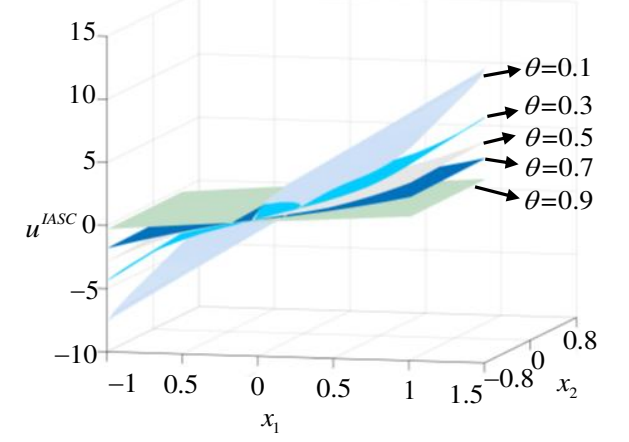

(c)

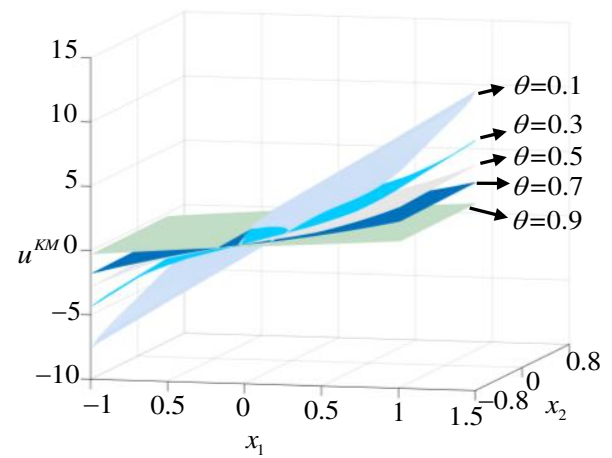

(d)

Fig. 3. The output surface of IT2 FC with, (a) use of NT TR, (b) use of WM TR, (c) use of IASC TR, (d) use of KM TR. The rule consequents $\left[\alpha_{1}, \beta_{1}\right]=[-15,-2],\left[\alpha_{2}, \beta_{2}\right]=[-10,-1]$,

$$
\left[\alpha_{3}, \beta_{3}\right]=[6,11],\left[\alpha_{4}, \beta_{4}\right]=[7,16] \text {. }
$$




\section{EFFECTS OF USING PRODUCT OPERATOR INSTEAD OF THE MIN() OPERATOR ON THE PROPOSED PROPERTIES}

The most widely used fuzzy operators in the fuzzy control are the $\min ()$ operator and the product operator, which affect the control performance of the IT2 FC, by providing the firing intervals. In the case of the $\min ()$ operator, the firing $\left[\alpha_{3}, \beta_{3}\right]=[6,11]_{\text {intervals are obtained as shown in (2), while (1) }}$ provides the firing intervals, in case of the product operator being used.

It should be noted that, although the product fuzzy operator is used to obtain firing intervals, in the proof of (Zhou et al., 2019), the conditions $0 \leq \underline{f}_{i}^{*}(\mathbf{x}, \theta) \leq \bar{f}_{i}^{*}(\mathbf{x}, \theta) \leq 1$, $\partial \underline{f}_{i}^{*} / \partial \theta_{i} \leq 0$ and $\partial \bar{f}_{i}^{*} / \partial \theta_{i} \geq 0$ still hold true. The entire proof process does not use the value of the firing intervals, so it does not affect the conclusions stated in (Zhou et al., 2019), i.e., $\left|\Delta u^{K M}(\mathbf{x}, \theta)\right|$

still approaches 0 as the FOUs increase and it actually becomes 0 , when the FOUs reach their maximum. Subsections $A, B$, and $C$ of Section III show that, the values of the firing intervals are also not used in the proof process, so the above properties are still true, even when the $\min ()$ operator is replaced by the product operator.

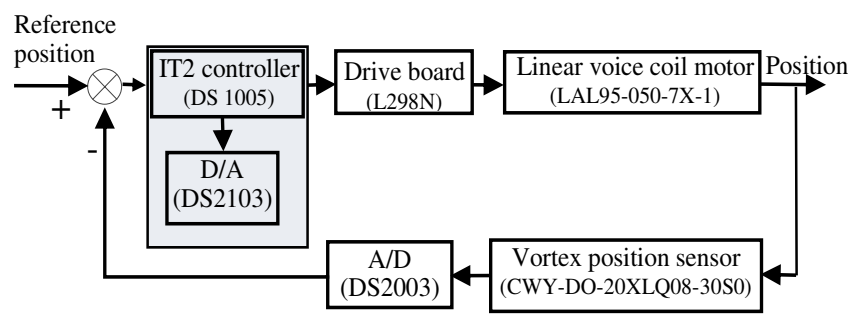

Fig. 4. Flow diagram of real-time IT2 Mamdani fuzzy control system.

\section{EXPERIMENT OF REAL-TIME FUZZY SYSTEM}

In order to test whether the response of the IT2 Mamdani FC, using different TRs, is consistent with the theoretical analysis, an IT2 fuzzy control system is designed, with all the configurations in Section II. In this real-time control system (Fig. 4), the controlled object is a linear voice coil motor (Model: LAL 950-050 model, SMAC Corp). Such motors are commonly used in high-acceleration and high-precision control systems, such as optical and chip mounting equipment. The nonlinear input-output relationship of the motor (Zhou \& Wang et al., 2019) and other nonlinear elements (such as sensors, D/A module and A/D module) constitute a highly nonlinear system, suitable for testing IT2 FCs. Other hardware in the experimental system includes a dSPACE DS 1005 processor and a Lenovo computer, using an $17-2600 \mathrm{KCPU}$ at $3.40 \mathrm{GHz}$ and $8.00 \mathrm{~GB}$ memory.

In the experiment, it is set $N=2$, the domain of the input variable $x_{1}$ is $[-0.25,0.25]$, the domain of $x_{2}$ is $[-10,10]$ and the membership functions are: $\bar{\mu}_{A_{1}^{k_{1}}}=-(1-\theta) x_{1} / 0.5+(1+\theta) / 2$, $\underline{\mu}_{A_{1}^{k_{1}}}=-(1-\theta) x_{1} / 0.5+(1-\theta) / 2, \bar{\mu}_{A_{1}^{k_{2}}}=(1-\theta) x_{1} / 0.5+(1+\theta) / 2$,
$\underline{\mu}_{A_{1}^{k_{2}}}=(1-\theta) x_{1} / 0.5+(1-\theta) / 2, \bar{\mu}_{A_{2}^{k_{1}}}=-(1-\theta) x_{2} / 20+(1+\theta) / 2$, $\underline{\mu}_{A_{1}^{k_{1}}}=-(1-\theta) x_{2} / 20+(1-\theta) / 2, \bar{\mu}_{A_{2}^{k_{2}^{2}}}=(1-\theta) x_{2} / 20+(1+\theta) / 2$, $\underline{\mu}_{A_{2}^{k_{2}}}=(1-\theta) x_{2} / 20+(1-\theta) / 2$. The four corresponding fuzzy rules are defined as:

IF $x_{1}$ is $A_{1}^{k_{1}}$ and $x_{2}$ is $A_{2}^{k_{1}}$ THEN $u$ is -0.35 Rule 1

IF $x_{1}$ is $A_{1}^{k_{1}}$ and $x_{2}$ is $A_{2}^{k_{2}}$ THEN $u$ is -0.15 Rule 2

IF $x_{1}$ is $A_{1}^{k_{2}}$ and $x_{2}$ is $A_{2}^{k_{1}}$ THEN $u$ is 0.15 Rule 3

IF $x_{1}$ is $A_{1}^{k_{2}}$ and $x_{2}$ is $A_{2}^{k_{2}}$ THEN $u$ is 0.35 Rule 4

The other configurations of the IT2 FC are consistent with those listed in Section II. The four TRs are used 10 times in sequence in real-time control system, in order to obtain more generic control response results.

Figs. 5-8 show the motor position response, when the IT2 fuzzy control system uses the respective TRs. The figures show

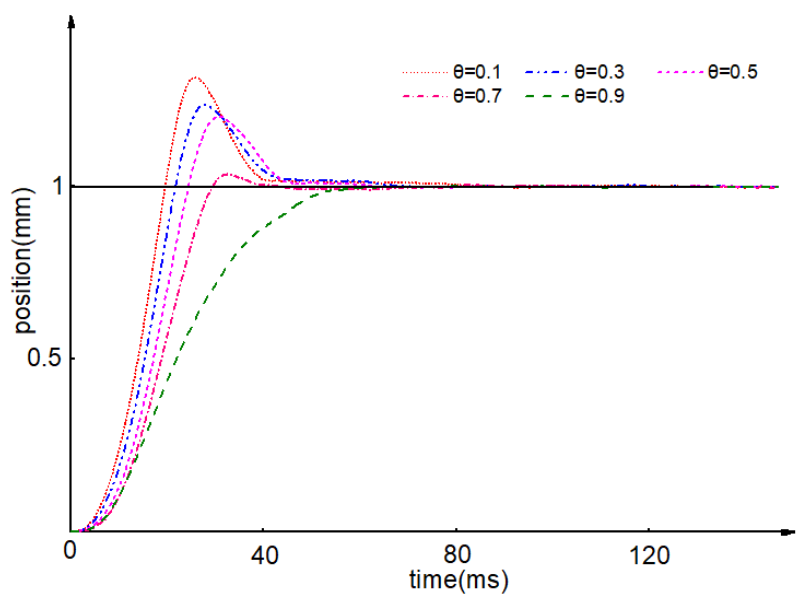

Fig. 5. Real-time position response of the motor when using NT TR.

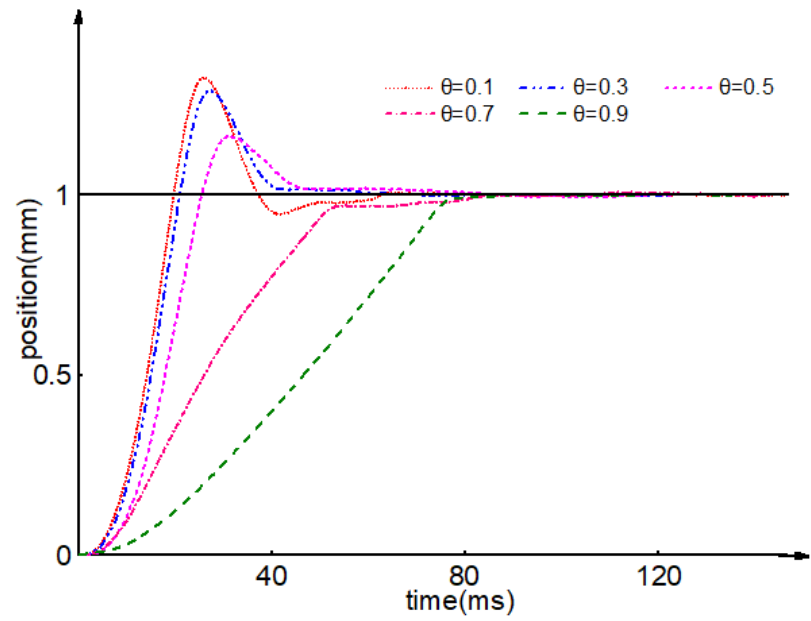

Fig. 6. Real-time position response of the motor when using WM TR 


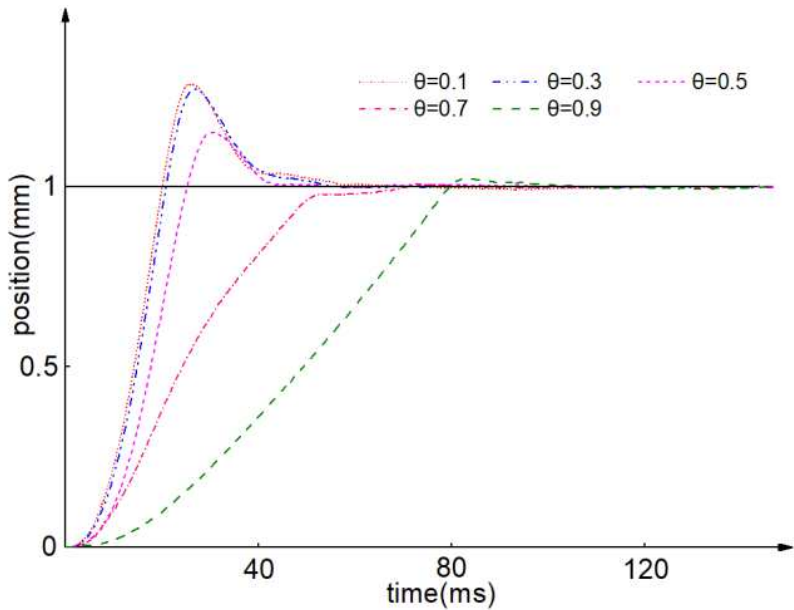

Fig. 7. Real-time position response of the motor when using IASC TR.

that, when identical FOUs are used, although the position curve of the motor is different, the position response of the motor gradually slows down, while the overshoot grows smaller, the setting time becomes longer. Moreover, the rule consequents are symmetrically configured about 0 , that is, the output of the controller gradually tends to the 0 plane. This occurrence is consistent with the theoretical analysis that shows the output of

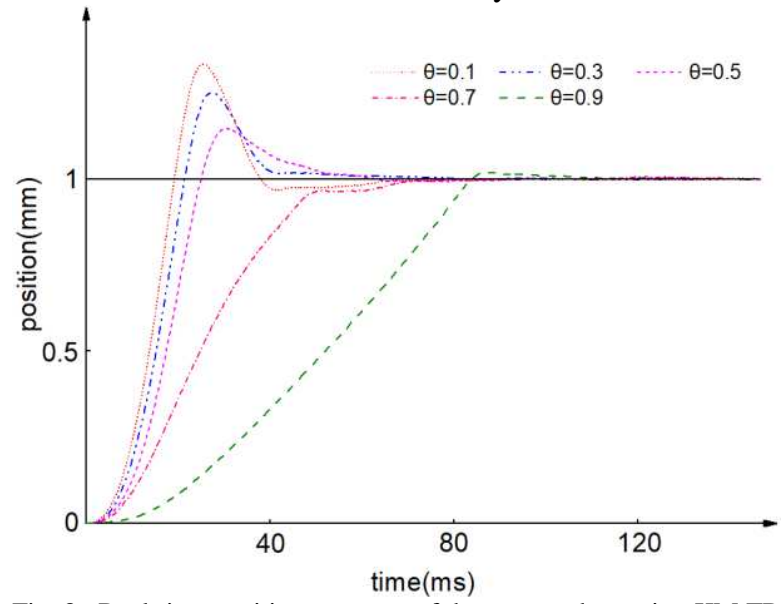

Fig. 8. Real-time position response of the motor when using KM TR.

the controller gradually tending to the constant plane, as the FOUs increase. In previous study presented in (Zhou \& Ying et al., 2019), it was demonstrated that, when the FOUs of the input fuzzy sets increased, the output of the IT2 Mamdani FC, using the KM TR, gradually tended to the constant controller, and eventually became one. When the FOUs are larger, the ability of system to resist disturbance obviously deteriorates and recovery time becomes longer. Therefore, one can speculate that when the FOUs are larger, the robustness of the IT2 fuzzy control system, using the NT TR, or WM TR, or IASC TR, will also be poor.

\section{CONCLUSION}

By studying the effects of increasing the FOUs on the output of IT2 Mamdani FC, using different TRs, it is proven that, whether KM TR, EKM TR, NT TR, WM TR or IASC TR is used, the controller is gradually converging to the behavior of a constant controller. When all the FOUs are at their maximum, the IT2 Mamdani FC using KM TR, EKM TR, NT or IASC TR becomes a constant controller, the controller using WM TR will approach the constant controller infinitely when FOUs are infinitely approaching their maximum.

The properties (1)-(7), proposed in this study, not only help to understand the role of the five widely used TRs in the IT2 FC, but also facilitate the analysis of the effects of increasing FOUs, while they can also provide guidance for the design of FOUs. Regardless of whether KM TR, EKM TR, NT TR, WM TR or IASC TR is used, the IT2 Mamdani FC, when using larger FOUs, will produce an output similar to the constant; that is, the fluctuation of the input variables have a limited impact on the output, while the ability of IT2 FC to deal with system uncertainties will deteriorate. When the FOUs are maximum (or infinitely approaching their maximum), the output of IT2 Mamdani controller is a constant (or approximates a constant infinitely), which is no longer a function of the input variables and the superiority of the FC disappears. In addition, according to the published study (Zhou \& Ying et al., 2019), properties (1)-(7) can be extended to the IT2 TS FC, but as the FOUs increase, this controller gradually tends to be a piecewise linear controller and actually becomes a piecewise linear controller when the FOUs are at their maximum.

Ethical approval This article does not contain any studies with human participants or animals performed by any of the authors. Funding This work was supported in part by the National Key R\&D Program of China [grant numbers 2017YFB1104800]; the National Natural Science Foundation of China [grant numbers 51975590].

Conflict of interests The authors declare that they have no known competing financial interests or personal relationships that could have appeared to influence the work reported in this paper.

Informed Consent All authors approved the final manuscript. Author contributions All authors contributed to the study conception and design. Material preparation, data collection and analysis were performed by [Chaolong Zhang], [Haibo Zhou], [Zhiqiang Li], [Xia Ju] and [Shuaixia Tan]. The first draft of the manuscript was written by [Chaolong Zhang] and all authors commented on previous versions of the manuscript. All authors read and approved the final manuscript.

\section{REFERENCES}

Chen, C., John, R., Twycross, J., \& Garibaldi, J. M. (2018). A Direct Approach for Determining the Switch Points in the Karnik - Mendel Algorithm. IEEE TRANSACTIONS ON FUZZY SYSTEMS, 26(2), 1079-1085. http://doi.org/10.1109/TFUZZ.2017.2699168

Chen, C., Wu, D., Garibaldi, J. M., John, R., Twycross, J., \& Mendel, J. M. (2018). A Comment on "A Direct Approach for Determining the Switch Points in the Karnik - Mendel Algorithm” . IEEE TRANSACTIONS ON FUZZY SYSTEMS, 26(6), 3905-3907. http://doi.org/10.1109/TFUZZ.2018.2865134

Chen, Y., \& Wang, D. (2018). Study on centroid type-reduction of general type-2 fuzzy logic systems with weighted enhanced Karnik - Mendel algorithms. SOFT COMPUTING, 22(4), 1361-1380. http://doi.org/10.1007/s00500-017-2938-3

Dongrui, U., \& Nie, M. (2011, 2011-01-01). Comparison and practical implementation of type-reduction algorithms for type-2 fuzzy sets and systems. Paper presented at the.

Du, X., \& Ying, H. (2010). Derivation and Analysis of the Analytical 
Structures of the Interval Type-2 Fuzzy-PI and PD Controllers [Article]. IEEE TRANSACTIONS ON FUZZY SYSTEMS, 18(4), 802-814. http://doi.org/10.1109/TFUZZ.2010.2049022

Hassan, S., Khosravi, A., \& Jaafar, J. (2015). The Impact of FOU Size and Number of MFs on the Prediction Performance of Interval Type-2 Fuzzy Logic Systems. In M. Ariff, M. N. Abdullah, S. Rahim, N. I. Arshad, J. Jaafar, \& I. A. Aziz (Eds.), (104-109). (Reprinted.

Huang, J., Ri, M., Wu, D., \& Ri, S. (2018). Interval Type-2 Fuzzy Logic Modeling and Control of a Mobile Two-Wheeled Inverted Pendulum. IEEE TRANSACTIONS ON FUZZY SYSTEMS, 26(4), 2030-2038. http://doi.org/10.1109/TFUZZ.2017.2760283

Karnik, N. N., Mendel, J. M., \& Liang, Q. (1999). Type-2 fuzzy logic systems. IEEE TRANSACTIONS ON FUZZY SYSTEMS, 7(6), 643-658. http://doi.org/10.1109/91.811231

Li, J., John, R., Coupland, S., \& Kendall, G. (2018). On Nie-Tan Operator and Type-Reduction of Interval Type-2 Fuzzy Sets. IEEE TRANSACTIONS ON FUZZY SYSTEMS, 26(2), 1036-1039. http://doi.org/10.1109/TFUZZ.2017.2666842

Long, Z., Xu, Y., \& Li, L. (2019). Analytical structures of interval type-2 fuzzy controllers using product AND operations. Advances in Mechanical Engineering, 11(5), 2072154226. http://doi.org/10.1177/1687814019851384

Melgarejo, M. (2007, 2007-01-01). A Fast Recursive Method to Compute the Generalized Centroid of an Interval Type-2 Fuzzy Set. Paper presented at the.

Mendel, J. M. (2013). On KM Algorithms for Solving Type-2 Fuzzy Set Problems. IEEE TRANSACTIONS ON FUZZY SYSTEMS, 21(3), 426-446. http://doi.org/10.1109/TFUZZ.2012.2227488

Mendel, J. M., Chimatapu, R., \& Hagras, H. (2020). Comparing the Performance Potentials of Singleton and Non-singleton Type-1 and Interval Type-2 Fuzzy Systems in Terms ofSculpting the State Space. IEEE TRANSACTIONS ON FUZZY SYSTEMS, 28(4), 783-794. http://doi.org/10.1109/TFUZZ.2019.2916103

Nie, M., \& Tan, W. W. (2008, 2008-01-01). Towards an efficient type-reduction method for interval type-2 fuzzy logic systems. Paper presented at the

Nie, M., \& Tan, W. W. (2012). Analytical Structure and Characteristics of Symmetric Karnik-Mendel Type-Reduced Interval Type-2 Fuzzy PI and PD Controllers [Article]. IEEE TRANSACTIONS ON FUZZY SYSTEMS, 20(3), 416-430. http://doi.org/10.1109/TFUZZ.2011.2174061

Roy, S. K., \& Maiti, S. K. (2020). Reduction methods of type-2 fuzzy variables and their applications to Stackelberg game. APPLIED INTELLIGENCE, 50(5), 1398-1415. http://doi.org/10.1007/s10489-019-01578-2

Runkler, T. A., Chen, C., \& John, R. (2018). Type reduction operators for interval type-2 defuzzification. INFORMATION SCIENCES, 467, 464-476. http://doi.org/10.1016/j.ins.2018.08.023

$\mathrm{Wu}$, D. (2012, 2012-01-01). An overview of alternative type-reduction approaches for reducing the computational cost of interval type-2 fuzzy logic controllers. Paper presented at the.

Wu, D. (2013). Approaches for Reducing the Computational Cost of Interval Type-2 Fuzzy Logic Systems: Overview and Comparisons. IEEE TRANSACTIONS ON FUZZY SYSTEMS, 21(1 ), 80-99. http://doi.org/10.1109/TFUZZ.2012.2201728

Wu, D., \& Mendel, J. M. (2009). Enhanced Karnik-Mendel Algorithms. IEEE TRANSACTIONS ON FUZZY SYSTEMS, 17(4), 923-934. http://doi.org/10.1109/TFUZZ.2008.924329

Wu, D., \& Mendel, J. M. (2019). Recommendations on designing practical interval type-2 fuzzy systems. ENGINEERING APPLICATIONS OF $\begin{array}{llll}\text { ARTIFICIAL INTELLIGENCE, } & \text { 85, }\end{array}$ http://doi.org/10.1016/j.engappai.2019.06.012

Wu, H., \& Mendel, J. M. (2002). Uncertainty bounds and their use in the design of interval type-2 fuzzy logic systems. IEEE TRANSACTIONS ON $\begin{array}{llll}\text { FUZZY } & \text { SYSTEMS, } & \text { 622-639. }\end{array}$ http://doi.org/10.1109/TFUZZ.2002.803496

Yip, C. M. T., Tan, W. W., \& Nie, M. (2019). On the difference in control performance of interval type-2 fuzzy PI control system with different FOU shapes. APPLIED SOFT COMPUTING, 76, 517-532. http://doi.org/10.1016/j.asoc.2018.12.039

Zhou, H., Wang, X., Zhang, Z., \& Duan, J. (2019). A Novel Thrust Force Test Method for a Class of Precision Noncontact Linear Motion Actuators. IEEE TRANSACTIONS ON INDUSTRIAL ELECTRONICS, 66(7), 5383-5391. http://doi.org/10.1109/TIE.2018.2868311

Zhou, H., Ying, H., \& Zhang, C. (2019). Effects of Increasing the Footprints of Uncertainty on Analytical Structure of the Classes of Interval Type-2 Mamdani and TS Fuzzy Controllers. IEEE TRANSACTIONS ON $\begin{array}{lrrr}\text { FUZZY SYSTEMS, } & 27(9) \\ \text { http://doi.org/10.1109/TFUZZ } & \text { 2019.2892354 }\end{array}$

Zhou, H., \& Ying, H. (2013). A Method for Deriving the Analytical Structure of a Broad Class of Typical Interval Type-2 Mamdani Fuzzy Controllers. IEEE TRANSACTIONS ON FUZZY SYSTEMS, 21(3), 447-458. http://doi.org/10.1109/TFUZZ.2012.2226891

Zhou, H., \& Ying, H. (2017). Deriving and Analyzing Analytical Structures of a Class of Typical Interval Type-2 TS Fuzzy Controllers. IEEE Transactions on Cybernetics, 47(9), 2492-2503. http://doi.org/10.1109/TCYB.2016.2570239 
Figures

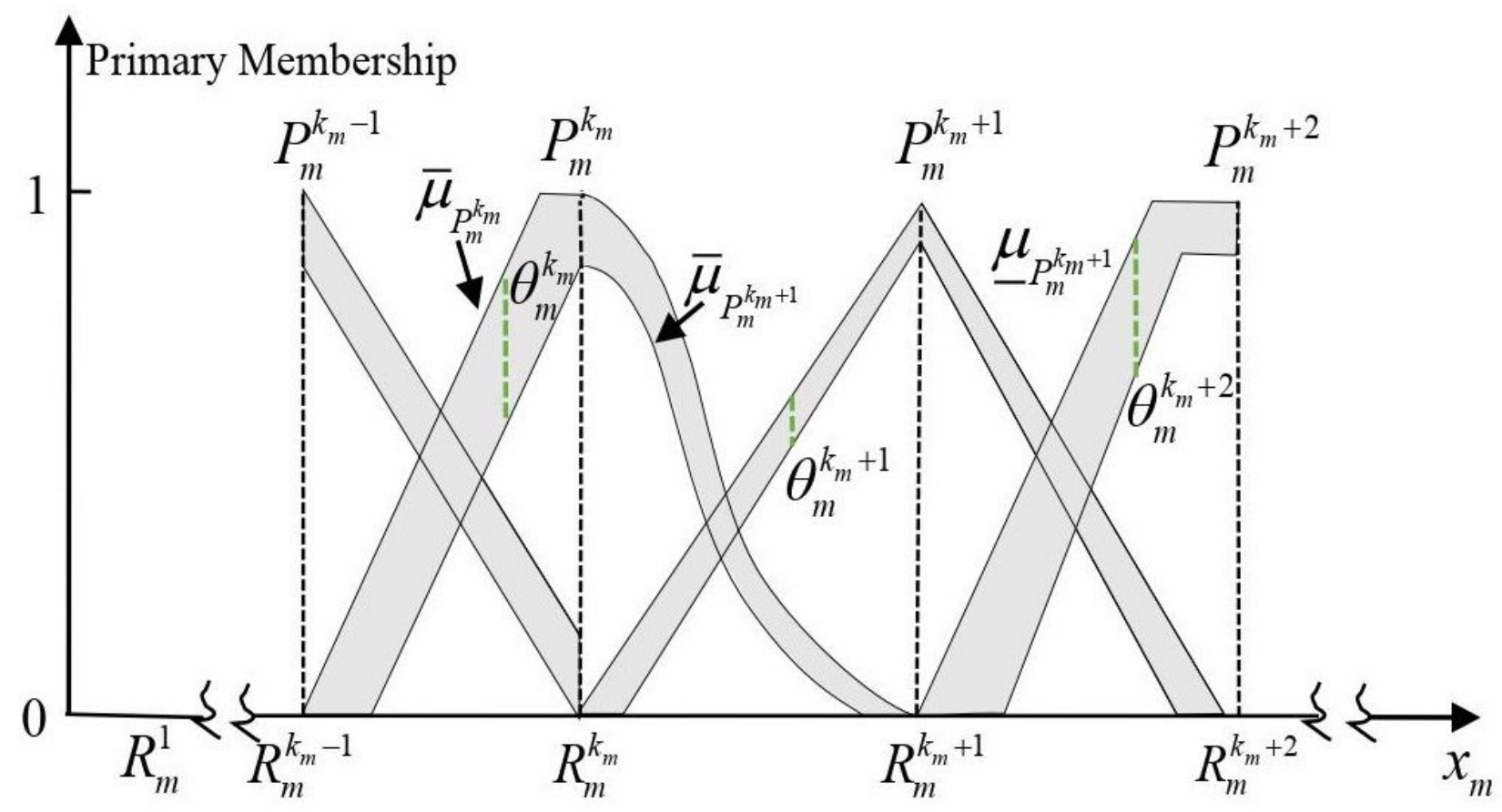

Figure 1

Example IT2 fuzzy sets for the input variable xm.

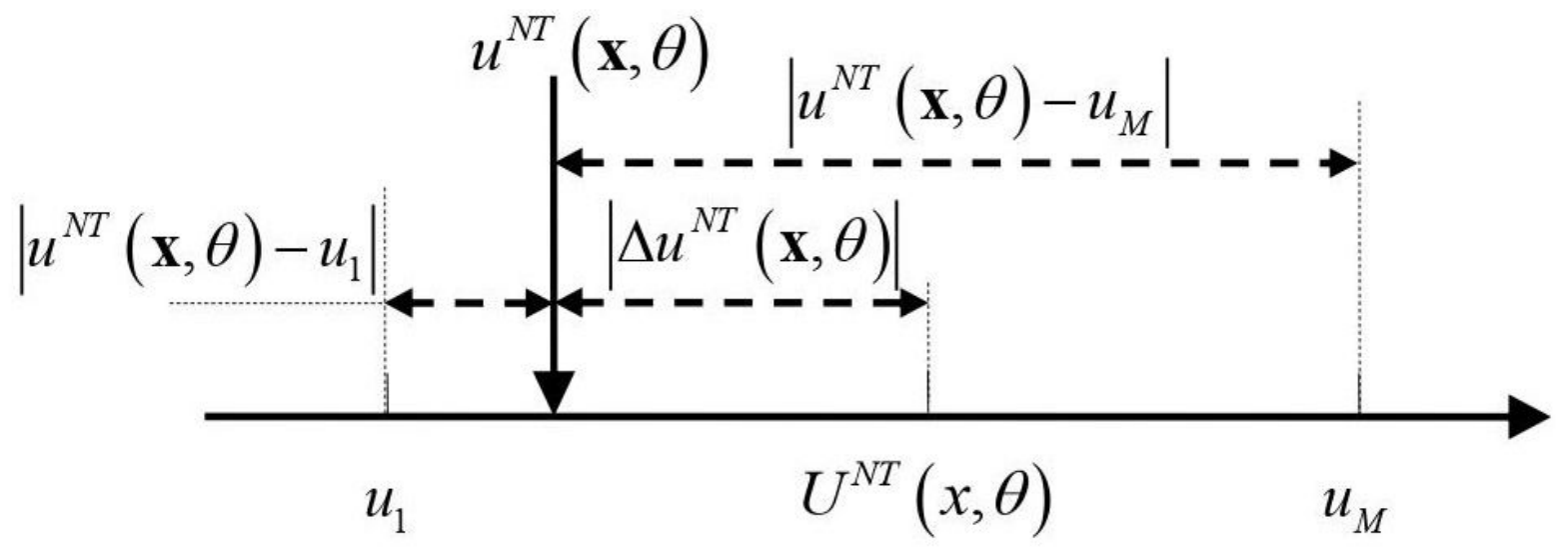

Figure 2

Please see the Manuscript PDF file for the complete figure caption 


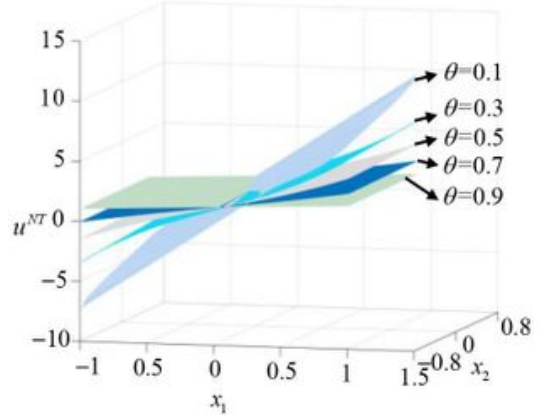

(a)

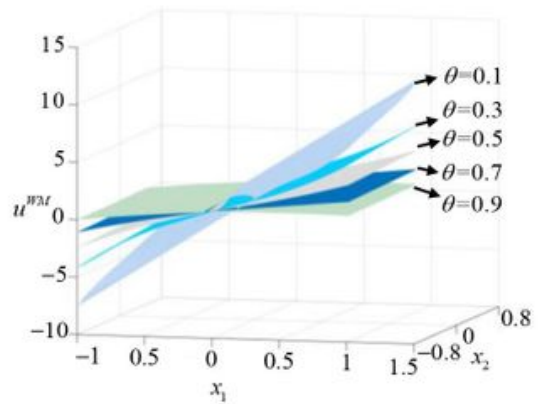

(b)

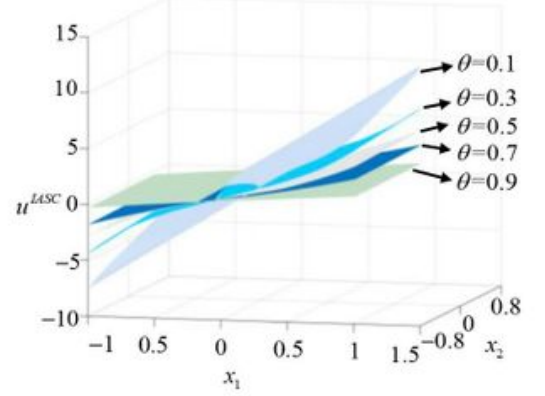

(c)

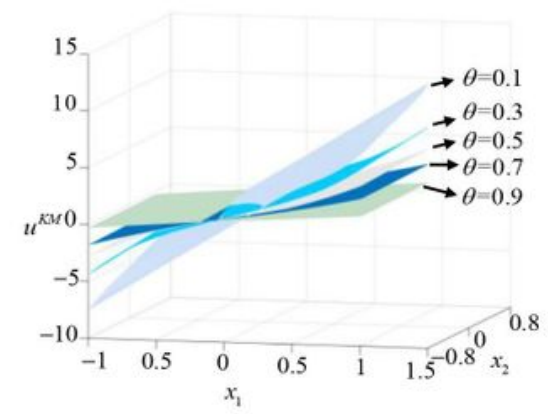

(d)

\section{Figure 3}

Please see the Manuscript PDF file for the complete figure caption 


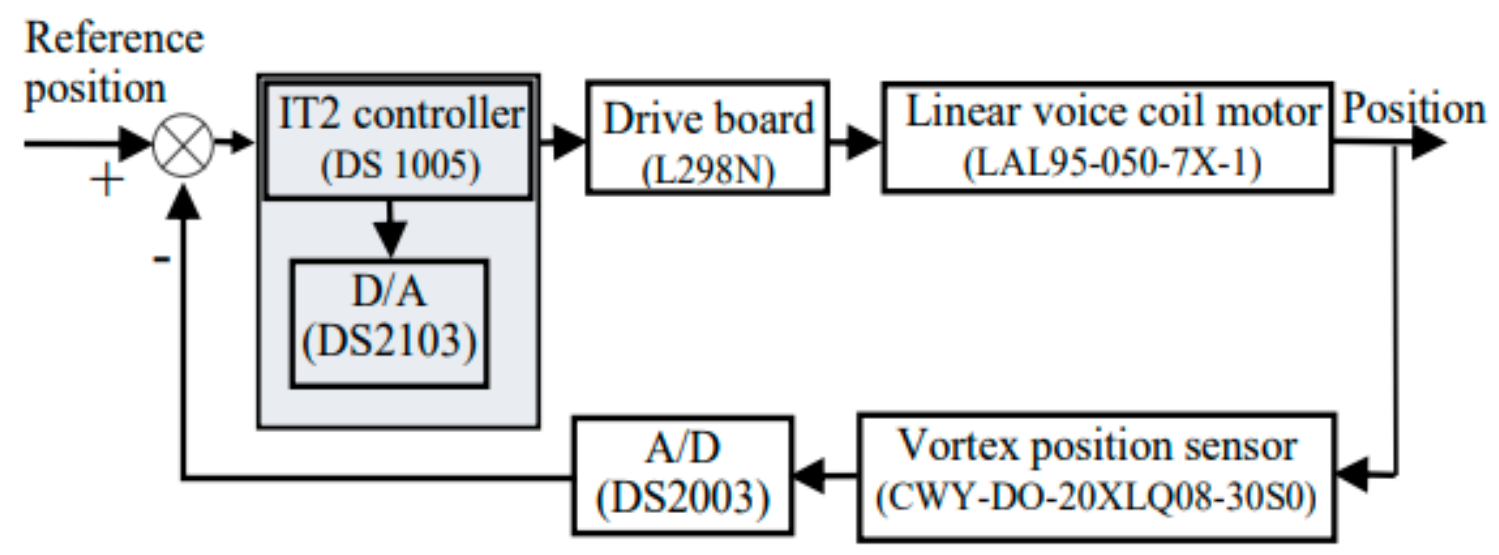

Figure 4

Flow diagram of real-time IT2 Mamdani fuzzy control system.

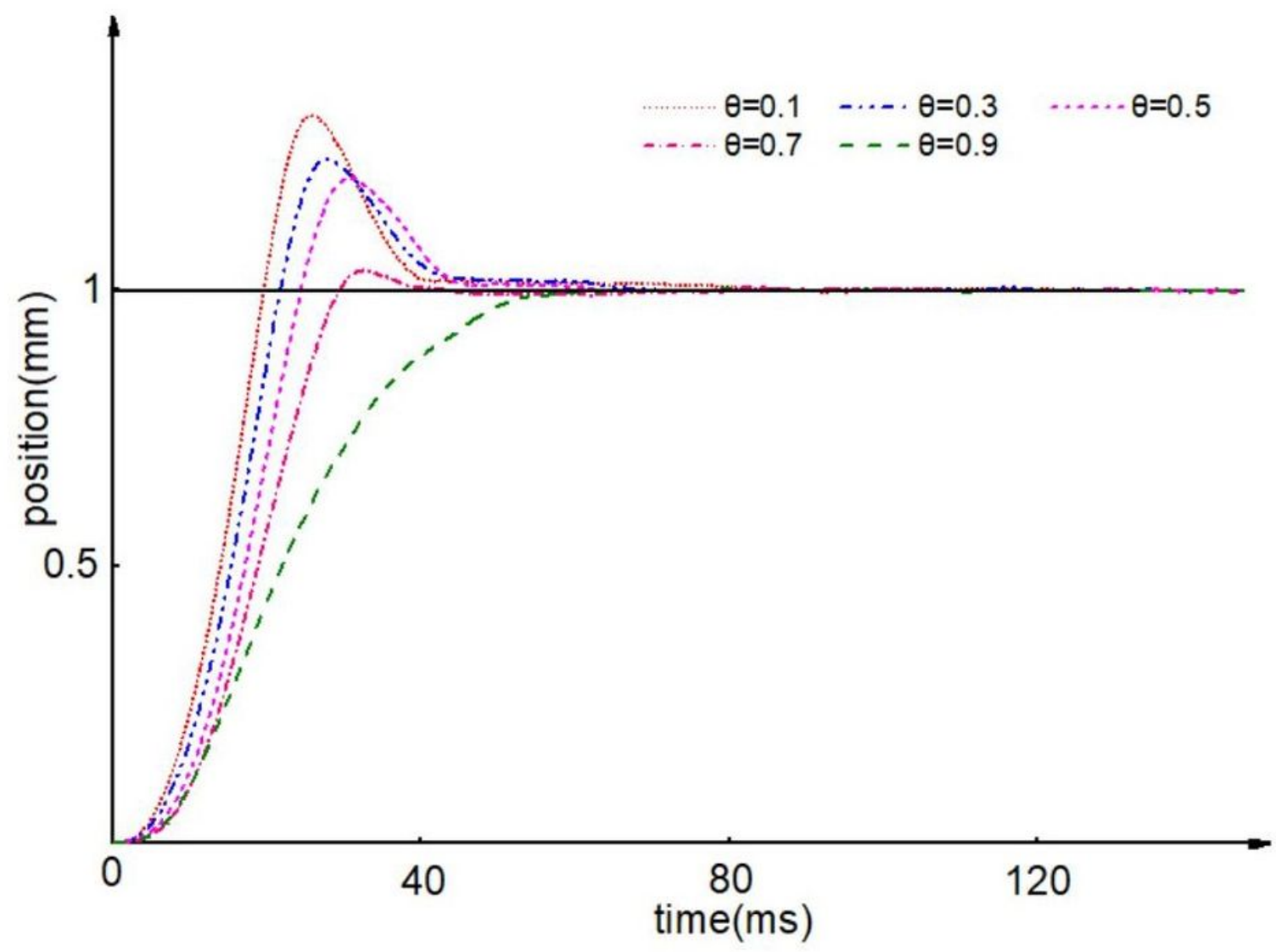

Figure 5

Real-time position response of the motor when using NT TR. 


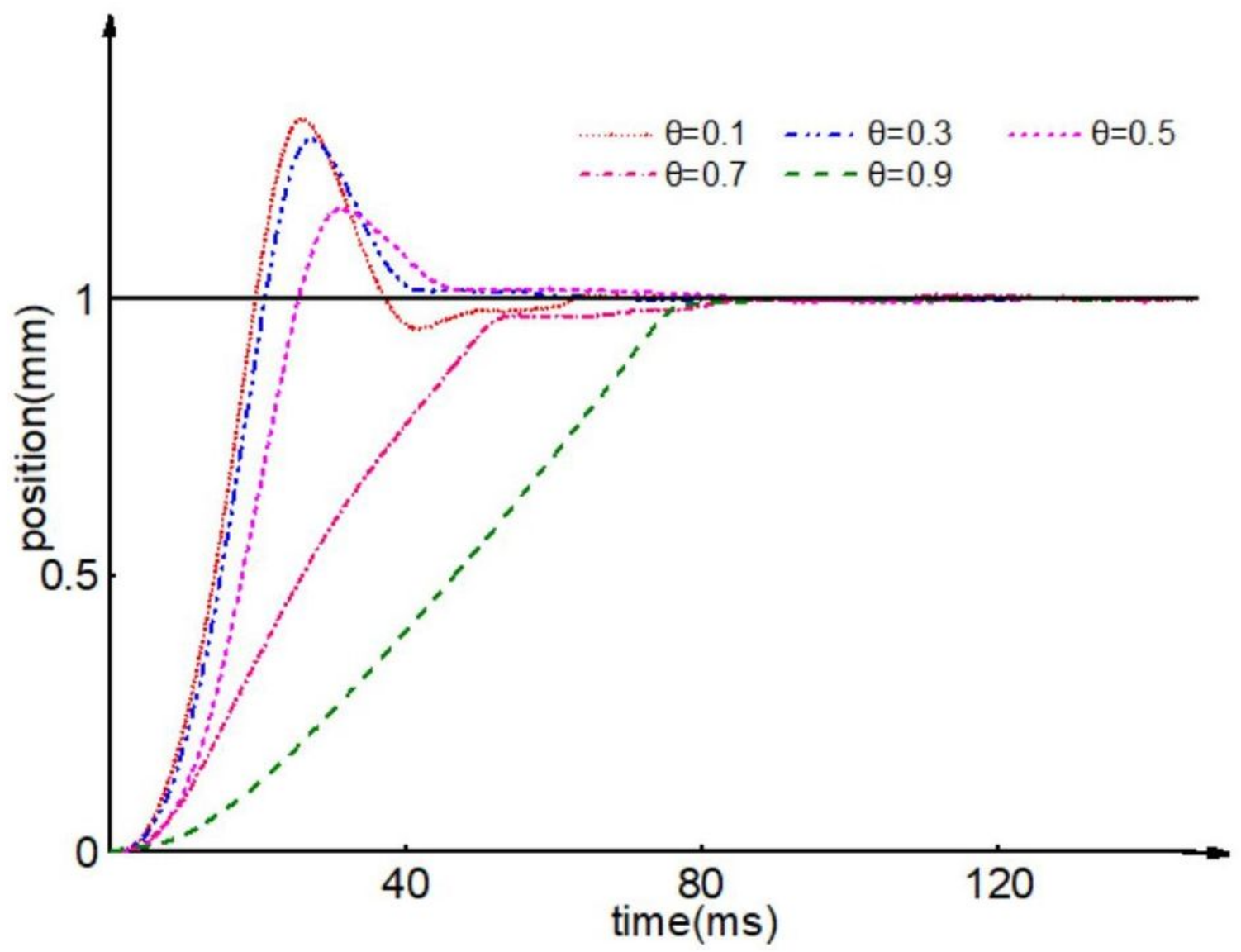

Figure 6

Real-time position response of the motor when using WM TR. 


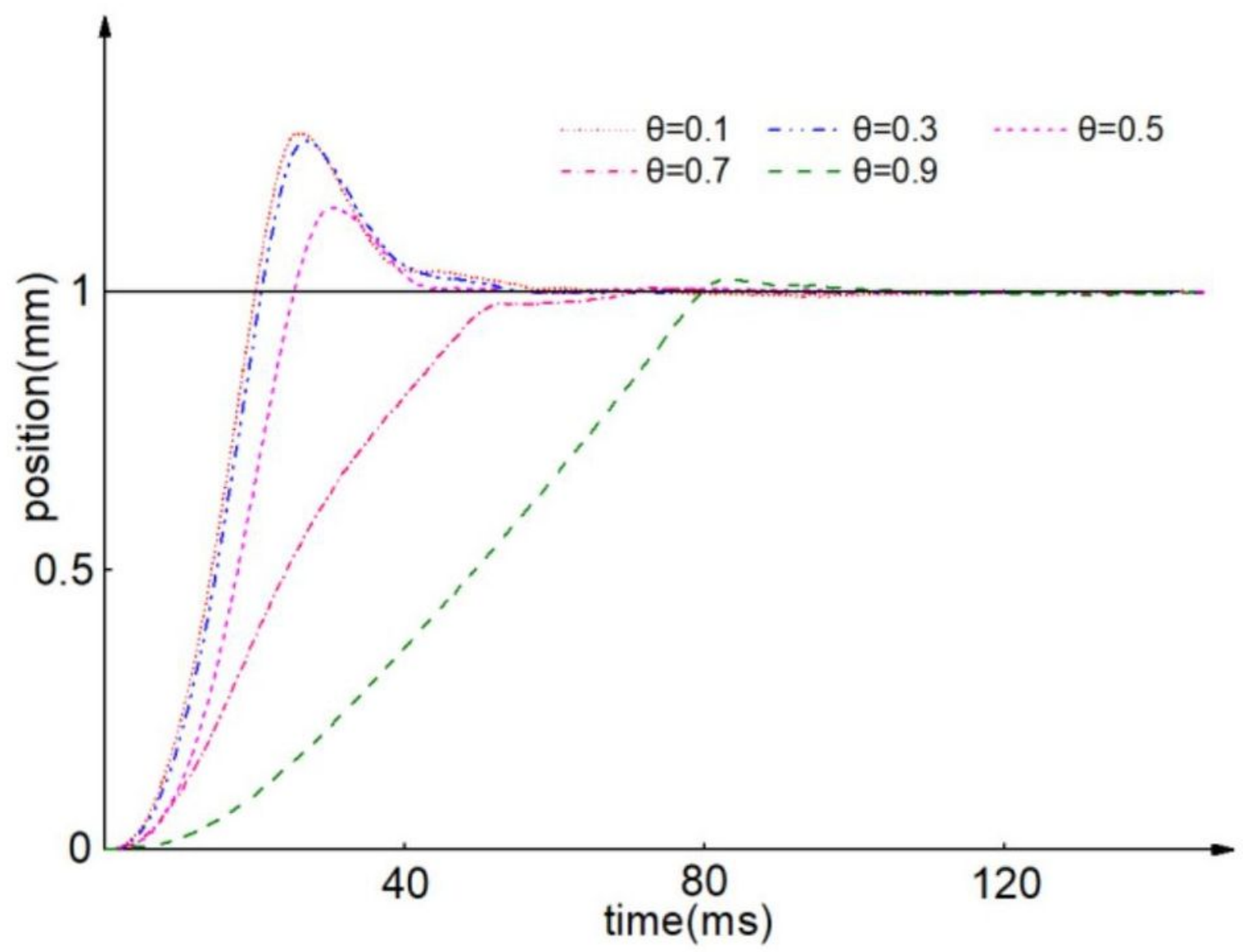

Figure 7

Real-time position response of the motor when using IASC TR. 


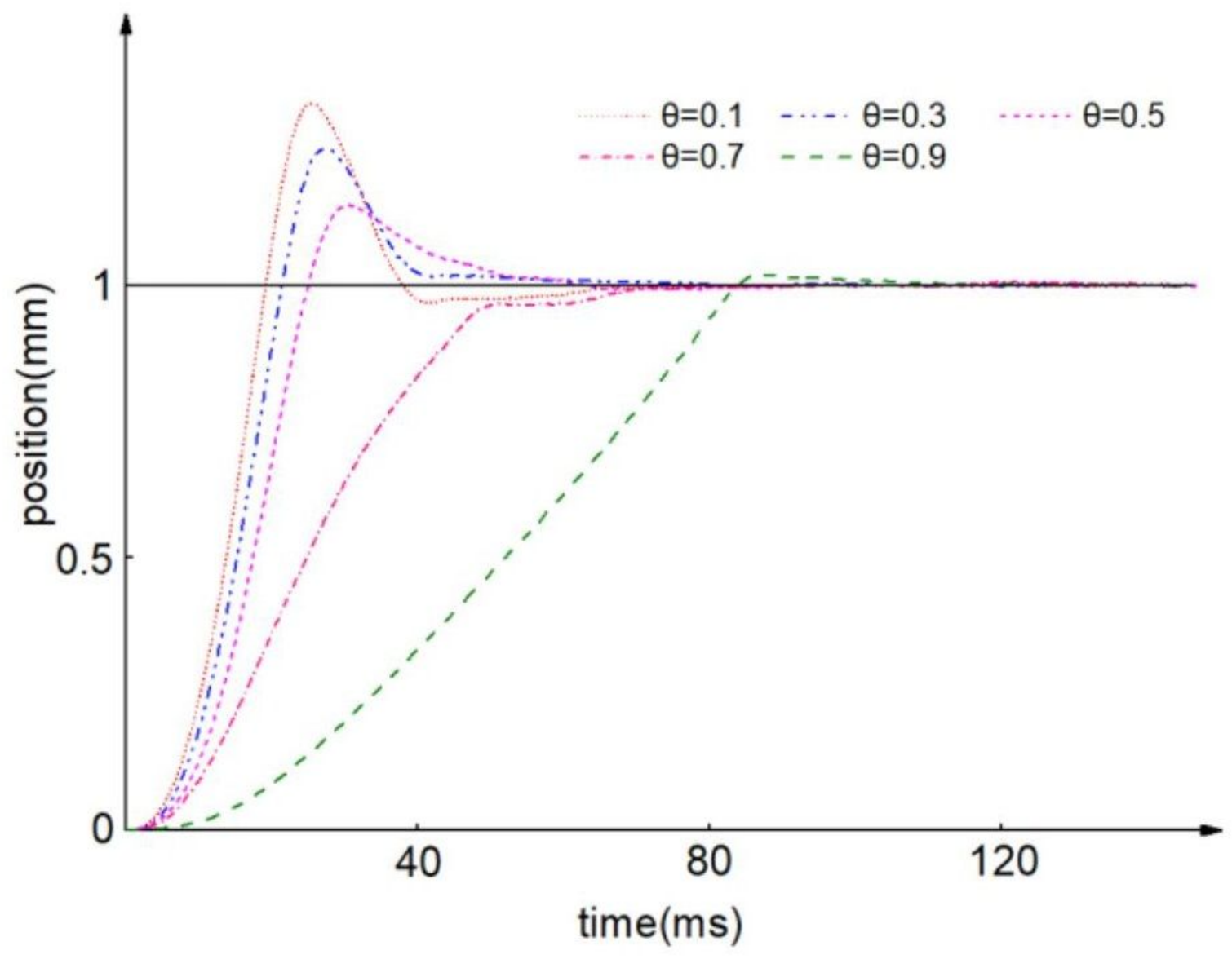

Figure 8

Real-time position response of the motor when using KM TR. 